\title{
Second-Order Multifunction Filters with Current Operational Amplifiers
}

\author{
David Kubanek and Kamil Vrba \\ Department of Telecommunications, Faculty of Electrical Engineering and Communication, \\ Brno University of Technology, Purkynova 118,612 00 Brno, Czech Republic \\ \{kubanek, vrbak\}@feec.vutbr.cz
}

\begin{abstract}
Novel second-order multifunction filters operating in current mode are described. Their structure is based on three current operational amplifiers connected in a feedback loop. These active elements are dual to the well-known operational amplifiers and are suitable for high-frequency signal processing. The designed filters offer low-pass, band-pass and high-pass transfer functions simultaneously. Computer simulation is carried out to prove the functionality of a filter variant. The influence of real properties of active elements is analyzed.
\end{abstract}

Keywords: active filter, current mode, current operational amplifier

\section{Introduction}

The demands on modern signal processing systems are still stricter. Particularly multimedia and other broadband signals must be processed by high-frequency electronic circuits. The development of new sub-micron integrated circuit technologies leads to the supply voltage lowering of and thus reducing the levels of processed voltage signals. The signal-to-noise ratio (SNR) decreases as well and that is why designers of recent electronic circuits increasingly use current signals to carry information. The SNR is better in current-mode circuits and their frequency bandwidth usually also increases.

Frequency filtering is one of the most often used operations in signal processing and frequency filters belong to the most widely used electronic circuits. This paper presents a design procedure of several second-order filter variants that employ current operational amplifiers as active building blocks, operate in current mode, and provide multiple transfer functions simultaneously.

\section{Current operational amplifier}

We have chosen the current operational amplifier (COA, also known as TCOA True Current Operational Amplifier) for the analog filter design. It is a relatively new building block for active circuits and several its variants were described in [1], [2], [3], [4], and [5] together with their possible internal structures designed in MOS or 
bipolar technologies. COA is a dual element to the well-known voltage feedback operational amplifier. It has a differential current input and infinite current gain. COA can be advantageously implemented in current mode active circuits, which process high-frequency analog signals. COA is usually connected in the negative feedback loop, which provides the equality of its input currents. The variant of COA with differential input and single output (DISO) as shown in Fig. 1 will be suitable for our design.

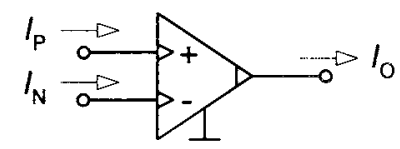

Fig. 1. Schematic symbol of COA

\section{Filter design}

The basic circuit for the filter design is shown in Fig. 2. It consists of three COAs connected in a global negative feedback loop. Each amplifier also has its local feedback loop formed by a passive one-port element and the circuit has three current inputs and three current outputs [6].

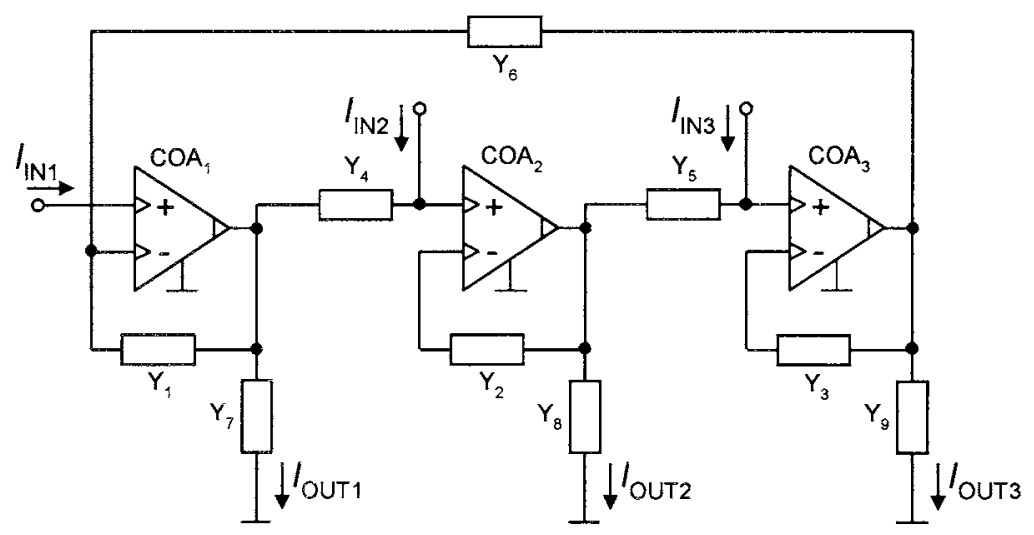

Fig. 2. The chosen general circuit with COAs

The proposed general network has the following characteristic polynomial (CP):

$$
D=Y_{1} Y_{2} Y_{3}+Y_{4} Y_{5} Y_{6} .
$$

Here $Y_{i}(i=1,2,3,4,5,6)$ are admittances of one-port elements used in the network in question. Let us divide these elements into two groups: the first one includes elements with admittances $Y_{1}, Y_{2}, Y_{3}$, the second one the elements with admittances $Y_{4}, Y_{5}, Y_{6}$. If arbitrary two admittances in the first group are $s C_{1}$ and $s C_{2}$ 
and the remaining admittance equals $G_{1}$, and if arbitrary one admittance in the second group is chosen as $\left(s C_{3}+G_{4}\right)$ and the remaining admittances are then denoted as $G_{2}$ and $G_{3}$, then we obtain the CP in the form:

$$
D(s)=s^{2} C_{1} C_{2} G_{1}+s C_{3} G_{2} G_{3}+G_{2} G_{3} G_{4} .
$$

We get the same $\mathrm{CP}$ if we interchange the groups above. If we skip the identical variants that arise due to the cyclic permutation, we can summarize the possible types of admittances in Tab. 1. We obtained 6 circuits (denoted by A, B, .. F), which are suitable for our purpose.

Table 1. Possible values of admittances

\begin{tabular}{|c|c|c|c|c|c|c|}
\hline Variant & $Y_{1}$ & $Y_{2}$ & $Y_{3}$ & $Y_{4}$ & $Y_{5}$ & $Y_{6}$ \\
\hline $\mathrm{A}$ & $s C_{1}$ & $s C_{2}$ & $G_{1}$ & $s C_{3}+G_{4}$ & $G_{2}$ & $G_{3}$ \\
\hline $\mathrm{B}$ & $G_{1}$ & $s C_{2}$ & $s C_{1}$ & $s C_{3}+G_{4}$ & $G_{2}$ & $G_{3}$ \\
\hline $\mathrm{C}$ & $s C_{1}$ & $G_{1}$ & $s C_{2}$ & $s C_{3}+G_{4}$ & $G_{2}$ & $G_{3}$ \\
\hline $\mathrm{D}$ & $s C_{3}+G_{4}$ & $G_{2}$ & $G_{3}$ & $s C_{1}$ & $s C_{2}$ & $G_{1}$ \\
\hline $\mathrm{E}$ & $s C_{3}+G_{4}$ & $G_{2}$ & $G_{3}$ & $G_{1}$ & $s C_{2}$ & $s C_{1}$ \\
\hline $\mathrm{F}$ & $s C_{3}+G_{4}$ & $G_{2}$ & $G_{3}$ & $s C_{1}$ & $G_{1}$ & $s C_{2}$ \\
\hline
\end{tabular}

All the possible current transfer functions of the network depicted in Fig. 2 have the form as seen in Tab. 2 .

Table 2. Transfer functions of the network in Fig. 2

\begin{tabular}{|c|c|c|}
\hline$\frac{I_{\mathrm{OUT1}}}{I_{\mathrm{IN} 1}}=\frac{Y_{2} Y_{3} Y_{7}}{D}$ & $\frac{I_{\mathrm{OUT} 2}}{I_{\mathrm{IN} 1}}=\frac{Y_{3} Y_{4} Y_{8}}{D}$ & $\frac{I_{\mathrm{OUT} 3}}{I_{\mathrm{IN} 1}}=\frac{Y_{4} Y_{5} Y_{9}}{D}$ \\
\hline$\frac{I_{\mathrm{OUT1}}}{I_{\mathrm{IN} 2}}=-\frac{Y_{5} Y_{6} Y_{7}}{D}$ & $\frac{I_{\mathrm{OUT} 2}}{I_{\mathrm{IN} 2}}=\frac{Y_{1} Y_{3} Y_{8}}{D}$ & $\frac{I_{\mathrm{OUT} 3}}{I_{\mathrm{IN} 2}}=\frac{Y_{1} Y_{5} Y_{9}}{D}$ \\
\hline$\frac{I_{\mathrm{OUT1}}}{I_{\mathrm{IN} 3}}=-\frac{Y_{2} Y_{6} Y_{7}}{D}$ & $\frac{I_{\mathrm{OUT2}}}{I_{\mathrm{IN} 3}}=-\frac{Y_{4} Y_{6} Y_{8}}{D}$ & $\frac{I_{\mathrm{OUT} 3}}{I_{\mathrm{IN} 3}}=\frac{Y_{1} Y_{2} Y_{9}}{D}$ \\
\hline
\end{tabular}

It is evident that for each of 6 circuit variants (see Tab. 1) 9 current transfer functions can be calculated, i.e. a total of 54 transfer functions. Let us choose the admittances at the outputs conductive: $Y_{7}=G_{\text {OUT1 }}, Y_{8}=G_{\text {OUT2 }}, Y_{9}=G_{\text {OUT3 }}$. Using the computed functions we can select 4 networks, which can be used as multifunction filters. It means that they operate as low-pass (LP), band-pass (BP) and high-pass (HP) filters simultaneously. All these networks have one input and three outputs or three inputs and one output. They are summarized with their transfer functions in Tab. 3 . 
Table 3. Transfer functions of multifunction filters

\begin{tabular}{|c|c|c|c|}
\hline \multirow{2}{*}{$\begin{array}{l}\text { Variant } \\
\text { (Tab. 1) }\end{array}$} & \multicolumn{3}{|c|}{ Transfer functions } \\
\hline & LP & BP & $\mathrm{HP}$ \\
\hline B & $\frac{I_{\mathrm{OUT} 1}}{I_{\mathrm{IN} 2}}=-\frac{G_{2} G_{3} G_{\mathrm{OUT} 1}}{D(s)}$ & $\frac{I_{\mathrm{OUT} 1}}{I_{\mathrm{IN} 3}}=-\frac{s C_{1} G_{3} G_{\text {OUT1 }}}{D(s)}$ & $\frac{I_{\mathrm{OUT} 1}}{I_{\mathrm{IN} 1}}=\frac{s^{2} C_{1} C_{2} G_{\mathrm{OUT} 1}}{D(s)}$ \\
\hline $\mathrm{C}$ & $\frac{I_{\text {OUT1 }}}{I_{\text {IN } 2}}=-\frac{G_{2} G_{3} G_{\text {OUT1 }}}{D(s)}$ & $\frac{I_{\text {OUT3 }}}{I_{\mathrm{IN} 2}}=\frac{s C_{1} G_{2} G_{\text {OUT3 }}}{D(s)}$ & $\frac{I_{\mathrm{OUT} 2}}{I_{\mathrm{IN} 2}}=\frac{s^{2} C_{1} C_{2} G_{\mathrm{OUT} 2}}{D(s)}$ \\
\hline $\mathrm{D}$ & $\frac{I_{\mathrm{OUT} 1}}{I_{\mathrm{IN} 1}}=\frac{G_{2} G_{3} G_{\mathrm{OUT} 1}}{D(s)}$ & $\frac{I_{\mathrm{OUT2}}}{I_{\mathrm{IN} 1}}=\frac{s G_{1} G_{3} G_{\text {OUT2 }}}{D(s)}$ & $\frac{I_{\mathrm{OUT3}}}{I_{\mathrm{IN} 1}}=\frac{s^{2} C_{1} C_{2} G_{\mathrm{OUT3}}}{D(s)}$ \\
\hline E & $\frac{I_{\mathrm{OUT1}}}{I_{\mathrm{IN1}}}=\frac{G_{2} G_{3} G_{\mathrm{OUTI}}}{D(s)}$ & $\frac{I_{\mathrm{OUT1}}}{I_{\mathrm{IN} 3}}=-\frac{s C_{1} G_{3} G_{\mathrm{OUT} 1}}{D(s)}$ & $\frac{I_{\mathrm{OUT1}}}{I_{\mathrm{IN} 2}}=-\frac{s^{2} C_{1} C_{2} G_{\mathrm{OUT1}}}{D(s)}$ \\
\hline
\end{tabular}

\section{Influence of COA real properties on filter characteristics}

Let us choose the variant $\mathrm{C}$ as an example for further analyses. A frequencydependent model of COA as shown in Fig. 3 will be used for PSpice simulation. This model includes parasitic properties that can be expected in a real COA (unfortunately, no COA has been commercially manufactured yet). The controlled sources CCCS and VCCS in the model are frequency independent. The terminals + and - have internal series resistances of $5 \Omega$. A shunt resistance of $10 \mathrm{M} \Omega$ and a capacitance of $5 \mathrm{pF}$ are connected to the high-impedance node and determine the amplifier bandwidth. The simulated magnitude frequency characteristics of the designed multifunction filter are depicted in Fig. 4.

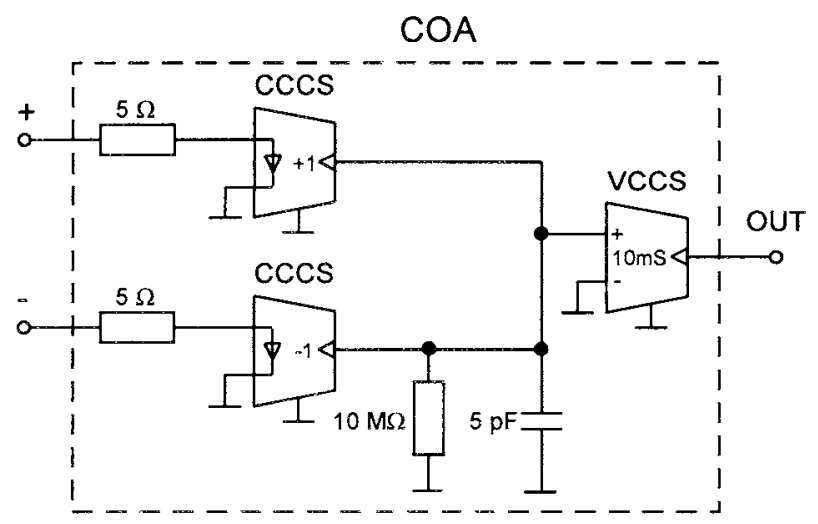

Fig. 3. The COA model used 


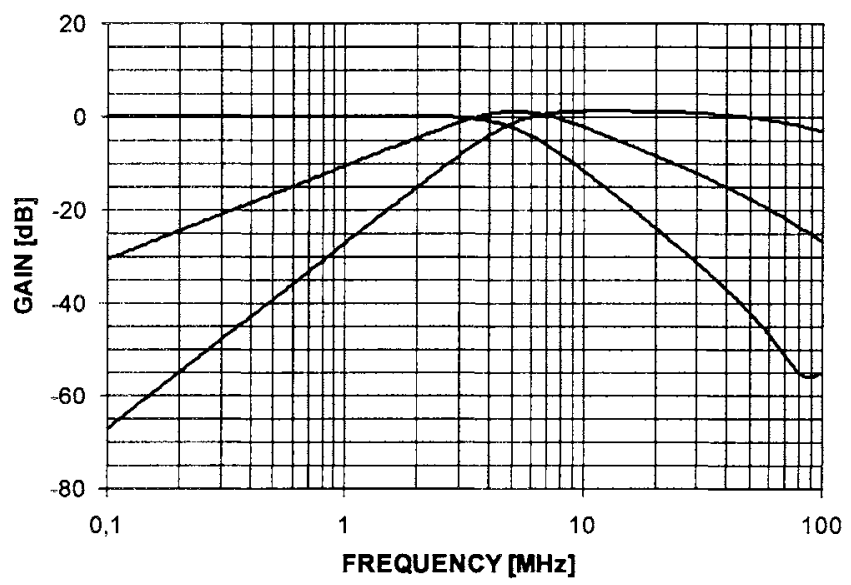

Fig. 4. The magnitude frequency characteristic of the designed filter

The magnitude frequency characteristics are correct up to ca $30 \mathrm{MHz}$. High-pass filter has a parasitic attenuation above this frequency, which is caused by non-zero input resistances and particularly by non-zero capacitance of the internal node in the COA model. This capacitance causes additional attenuation also at the low-pass and band-pass characteristics at high frequencies. The internal capacitance also causes the parasitic zero of the low-pass filter (apparent at about $90 \mathrm{MHz}$ in Fig. 4) and its higher values shift the zero to lower frequencies.

The resistance of the high-impedance node has an influence only on the band-pass and high-pass characteristic. It causes finite and constant attenuation at low frequencies, which are out of range of Fig. 4. Finite attenuation appears also at highfrequency parts of the low-pass and band-pass characteristics which are above $100 \mathrm{MHz}$ and thus also not apparent in Fig. 4. Causes of these effects will be investigated in detail in chapter 4.1.

We are looking for the replacement of COA by a commercially available element and if it is possible, we will present practically measured characteristics in the conference presentation.

\subsection{Analysis of finite attenuation in stop-band}

As mentioned in the last chapter, the real COA properties included in the model in Fig. 3 cause finite and constant attenuation in stop-bands of all LP, BP, and HP filters. Let us find relations for the transfer values in stop-bands. The parameters of filter passive elements and the COA input resistance $R_{\mathrm{TN}}$ can be expressed as a product or a ratio of geometric means $C, G$, and coefficients $m, n, p, z$ as shown in Tab. 4. This representation leads to the independent relations for the ideal pole frequency $\omega_{\mathrm{pi}}$ and the ideal pole quality factor $Q_{\mathrm{pi}}$ of the filter, see (3). 
Table 4. Substitution of filter component parameters

\begin{tabular}{|c|c|c|c|c|c|c|c|c|c|c|}
\hline$C_{1}$ & $C_{2}$ & $C_{3}$ & $G_{1}$ & $G_{2}$ & $G_{3}$ & $G_{4}$ & $G_{\text {OUT } 1}$ & $G_{\text {OUT2 }}$ & $G_{\text {OUT3 }}$ & $R_{\mathrm{IN}}$ \\
\hline$m C$ & $C / m$ & $n C$ & $G$ & $G$ & $G / p$ & $p G$ & $p G$ & $G$ & $n G /(m p)$ & $1 /(z G)$ \\
\hline
\end{tabular}

$$
\omega_{\mathrm{pi}}=\frac{G}{C} ; \quad Q_{\mathrm{pi}}=\frac{p}{n} .
$$

With the substitution according to Tab. 4 , it is easy to express the limits of transfer magnitudes in the stop-bands. They are summarized in Tab. 5, where $A_{0}$ is the lowfrequency gain of COA, which is equal to the product of the resistance of the highimpedance node and the transconductance of VCCS.

Table 5. Limits of transfers in stop-bands

\begin{tabular}{|c|c|c|c|}
\hline $\mathrm{P}$ & \multicolumn{2}{|c|}{ B } & I \\
\hline $\lim _{f \rightarrow \infty} K=\frac{p^{2}}{2 p z+p^{2}+3}$ & $\lim _{f \rightarrow 0} K=\frac{n}{A_{0} m}$ & $\lim _{f \rightarrow \infty} K=\frac{n}{2 p^{2} z^{2} m}$ & $\lim _{f \rightarrow 0} K=\frac{1}{A_{0}^{2}}$ \\
\hline
\end{tabular}

The values of $z$ and $A_{0}$ can be assumed much higher than $m, n, p$ in most cases. Then the high-frequency transfer of LP filter is indirect proportional to $z$ and thus it increases proportionally to the COA input resistance. The relation for the highfrequency transfer of BP has also $z$ in the denominator, but this transfer does not increase with the COA input resistance so dramatically thanks to the square. The lowfrequency transfers of BP and HP filters increase with reducing the low-frequency gain of COA, whereas the HP transfer increases less dramatically due to the square in the relation.

\section{Conclusions}

New second-order multifunction filters are described in the paper. Four unique variants were derived from a general basic circuit. They can operate as low-pass, band-pass and high-pass filters simultaneously. One of the variants was chosen for the computer simulation with frequency dependent COA model. It was proved that the filter operates correctly also at relatively high frequencies. It was investigated how the real properties of $\mathrm{COA}$ affect the functionality of the filter. The non-zero input resistances and internal node capacitance of the COA model mostly influence the characteristics. 
Acknowledgment. The paper has been prepared as a part of the solution of the Czech Republic Grant Agency projects 102/06/1383 and 102/07/P353.

\section{References}

1. Tomazou, C., Lidgey, F. J., Haigh D. G.: Analogue IC design: the current-mode approach. Peter Peregrinus Ltd, 1990

2. Bruun, E.: A differential-input, differential-output current mode operational amplifier. Int. J. Electronics, 1991, Vol. 71, No. 6, pp. 1047-1056

3. Kaulgerg, T.: A CMOS Current-Mode Operational Amplifier. Int. Journal of Solid-State Circuits, Vol. 28, No. 7, July 1993, pp. 849-852

4. Mucha, I.: Towards a true current operational amplifier. Proceedings of the ISCAS' 94 Conference, London, May 30-June 2, 1994, Vol. 5, pp. 389-392

5. Nagasaku, T., Hyogo, A., Sekine, K.: A Synthesis of a Novel Current-Mode Operational Amplifier. Analog Integrated Circuits and Signal Processing, 11, 1996, pp. 183-185

6. Cajka, J., Vrba, K., Kubanek, D.: Novel multifunction second-order filters which are electronically tunable. Proceedings of the Radioelektronika Conference, Brno, May 6-7, 2003, pp. 21-24

7. Ghausi, M. S., Laker, K. R.: Modern filter design. Prentice Hall, New Jersey, 1981 